International Journal of Managing Information Technology (IJMIT) Vol.11, No.2, May 2019

\title{
DeVEloping THE E-COMMERCE MODEL A CONSUMER TO CONSUMER USING BLOCKCHAIN NETWORK TECHNIQUE
}

\author{
${ }^{1}$ Samer Shorman, ${ }^{2}$ Mohammad Allaymoun, ${ }^{3}$ Omer Hamid \\ ${ }^{1}$ Department of Computer Science, Applied Science University, Kingdom of Bahrain \\ ${ }^{2,3}$ Administrative \& Financial Sciences, AMA International University, Kingdom of \\ Bahrain
}

\begin{abstract}
E-commerce has increased recently because of the development of the internet and has become a new concept that is applicable to trade transaction and services providing, using information technology. This is known as e-commerce thatis a means of communicating information $\square$ products or services through technical tools. This research proposed model is able to take advantage of Blockchain technology to develop e-commerce especially consumer toconsumer. The proposed model adds some advantages to ecommerce operations, and the possibility of developing them to reach a high percentage of profits by using blockchain technology which led to verify the information of products offered for sale. In addition, to the possibility of distributing feedback to all Blockchain users, through which it develops the mechanism of trust and cooperation between consumers, it is considered a reference point to explore the behaviour of commercial consumers which is stored in the data file of consumers. This model facilitates business processes between consumer andconsumer, eliminates the central role of large business companies in controlling and setting restrictions, and to the development and expansion of this type of trade.
\end{abstract}

\section{KEYWORDS}

Blockchain, Network, E-Commerce, Consumer To Consumer

\section{INTRODUCTION}

The increasing spread of information and communication technologies, specifically the Internet; the global business community has been able to move towards electronic commerce [1]. It is providing many new features such as the possibility of providing all goods on electronic platforms, providing detailed information of goods, about the products offered [2]. It has become a good environment for competition by traders to reach the satisfaction of consumers which measure the extent of consumer satisfaction by tracking the feedback of consumers [3]. The growth of the e-commerce economy is expected to become the fastest growing trading format at this time.

The consumer-to-consumer (C2C) method means of exchanging products and services between each other. Through an online market maker such as eBay, where consumer prepares the product for the market, it puts the product into auction or for sale. Moreover, the market makers providea search engine and product display capabilities so that traditional transactions between consumers 
International Journal of Managing Information Technology (IJMIT) Vol.11, No.2, May 2019

can be reduced to one another [4]. Thereby making it easier for consumers to explore goods, pay the price, and then deliver goods.

Despite the great proliferation of electronic commerce, it has its drawbacks. The most concern isthe centrality of market platforms and the size of the companies [5]. This has become a hindrance to creating new investment opportunities, putting obstacles for novice investors so that only central platforms should be dealt with. Sellers are often required to pay high commission fees, but they do not have multiple options if they want to reach a large audience because the ecommerce platforms have good confidence. Furthermore, fees can reach $20 \%$ in some cases [6]. There is also a credit card or payment processor fee needed for approval. For example, a fee is usually charged at about 3\%; all these additions must be included in the price and transferred to the consumer [7].

Some electronic platforms determine the buyer-seller connection. Traders are struggling to build a long-term relationship with consumers. Communication is often restricted or falls within strict criteria. It is therefore necessary to find an alternative environment that provides effective ecommerce thereby eliminating the centralization of large companies that restrict trade between consumers [8]. This research attempts to link the concept of Blockchain and its technologies to eliminate centralization in business dealings.

Blockchain is one of the options to electronic commerce, which based on buying and selling among consumers. Blockchain has become an important technology to resolve the ideal to provide confidence without the need for a third party centrally supervising the processes between the seller and the buyer, in order to verify the accuracy of data and information [9]. In addition to playing an important role in converting many of the traditional operations central to digital operations decentralized, without the needfor any party such as a bank, company, or government [10]. It is a mechanism based on the principle of the record of the consumers in this way, where all records distributed are to all participants (all for all). Recently, the Blockchain technique and some digital computing concepts were combined such as the Bitcoin digital currency [11].

Since the appearance of Blockchain, many professionals have tried to link them to commerce, through the creation of decentralized markets such as Open Bazaar, Syscoin, Particl, etc. and link it to payment systems using currency encoded in stores, such as Monetha, TenX, Plutus, and others [12]. While the focus has been on encrypted processes and linking them to Blockchain techniques, decentralized markets are trying to combine traditional trade and e-commerce.

This research focuses on the uses of the Blockchain technology to create a model for e-commerce so as to be an ideal solution to create a trustworthy and reliable environment betweenconsumers without the middle part and to create decentralized markets.

\section{Electronic COMMERCE}

E-commerce is the activity of online buying and selling products; E-commerce is based on technologies such as mobile application, electronic transformation, supply chain management, electronic marketing, transaction processing, electronic data exchange, inventory management systems, and automated data collection systems $[1,2,13]$.

E-commerce generally uses the internet as a part of the transaction. Although there are many other technologies used such as the e-mail [14]. E-commerce includesonlinepurchasing (book, 
International Journal of Managing Information Technology (IJMIT) Vol.11, No.2, May 2019

music (downloading music in a digital distribution such as the iTunes Store). Electronic commerce includes various types such as Business to Consumer (B2C), Business to Business (B2B), Consumer to Consumer (C2C), Consumer to Business (C2B), and more [15]. This study will concentrate on $\mathrm{C} 2 \mathrm{C}$.

\section{Consumer to Consumer (C2C)}

Consumer to Consumer (C2C) provides an innovative way to allow consumers to interact with each other by selling goods and services [16]. While traditional markets require physical interactions between parties where the consumer approaches the store to buy a product or a service, at its most basic level, C2C represents a good market environment where a consumer buys goods from another consumer using a third-party business or platform to facilitate the transaction [17]. C2C companies are a type of model that has emerged with e-commerce technology and a participatory economy that has contributed to improving the working environment of both client and trader. So the advantage lies for consumers that they benefit from product competition and they can often find goods that are hard to find elsewhere [18].

In addition, margins are high for vendors because of the small costs due to the absence of retailers or wholesalers. $\mathrm{C} 2 \mathrm{C}$ sites are easy to navigate because there is no need to visit the store. Consumers offer their products online and buyers come to them [4]. As in eBay case where items are shipped directly from seller to buyer.

C2C sites and similar platforms earn seller fees for displaying products for sale like Amazon, eBay, instead of promotional features and facilitating credit card transactions. Moreover, the $\mathrm{C} 2 \mathrm{C}$ market is expected to grow in the future because of its cost-effectiveness. The cost of using a third party is declining, and the amount of products for sale by consumers is rising steadily [16].

Retailers are an important business model because of the popularity of social media channels. These channels offer specific products already owned by consumers and increase demand, therefore increasing the accessibility to $\mathrm{C} 2 \mathrm{C}$ platforms.

However, $\mathrm{C} 2 \mathrm{C}$ has some problems, for example, non-quality control or payment guarantees. As in some cases, the credit card payments may not be supported, even though the emergence of PayPal and other payment systems over the years has helped streamline payments on $\mathrm{C} 2 \mathrm{C}$.

\section{BLOCKCHAIN}

Blockchain is a growing list of logscalled blocks, which are associated with the use of encryption. Each block contains encryption segmentation for the previous block, a time stamp, and transaction data (typically represented as tree root segmentation) [9].

Blockchain is a resistive data modification. It is an "open and distributed register and records transactions between two parties efficiently and in a verifiable manner." It is used as a ledger distributed among consumers [10].

Blockchain is typically managed by a peer-to-peer network, which is collectively committed to a protocol to communicate between nodes and verify new clusters. Once data is recorded in any given block, it cannot be changed retroactively without changing all the following blocks, 
International Journal of Managing Information Technology (IJMIT) Vol.11, No.2, May 2019

requiring compatibility in most networks. Although blockchain records are not changeable, block chains can be considered safe by design and is an embodiment of a distributed computing system. Accordingly, a decentralized consensus was adopted on the use of data blocks [17].

Nakamoto Satoshi invented Blockchain in 2008 to act as a general ledger for the bitcoin [18]. The invention of blockchain for bitcoin is made of the first digital currency to solve the problem of double spending without the need for a reliable authority or central server. The design has inspired other home applications [19], and blockchains that can be read by the public are widely used by cryptocurrency.

Blockchain is a payment method. It has been proposed specially for commercial uses. Many entities such as Computerworld have called for the marketing of such blockchains without an appropriate safety mode [20].

Blockchain technology provides private key encryption which is a powerful tool that provides the authentication requirements. The Owning of the private key is considered as a property. It also exempts a person from having to share more personal information than necessary to complete the exchange, making him vulnerable to hackers [21].

The special features of the blockchain ledgers thatare stored in many different locations, this protects the data from modification (Bitcoin is estimated to have more than 35,000 nodes in its own P2P network); it cannot be destroyed [22]. In addition, since many different and independent nodes are working on tracking the ledger, adjusting it in an unreliable way will not go away because all other contracts will not approve this transaction and will not add it to the ledger figure 1 .

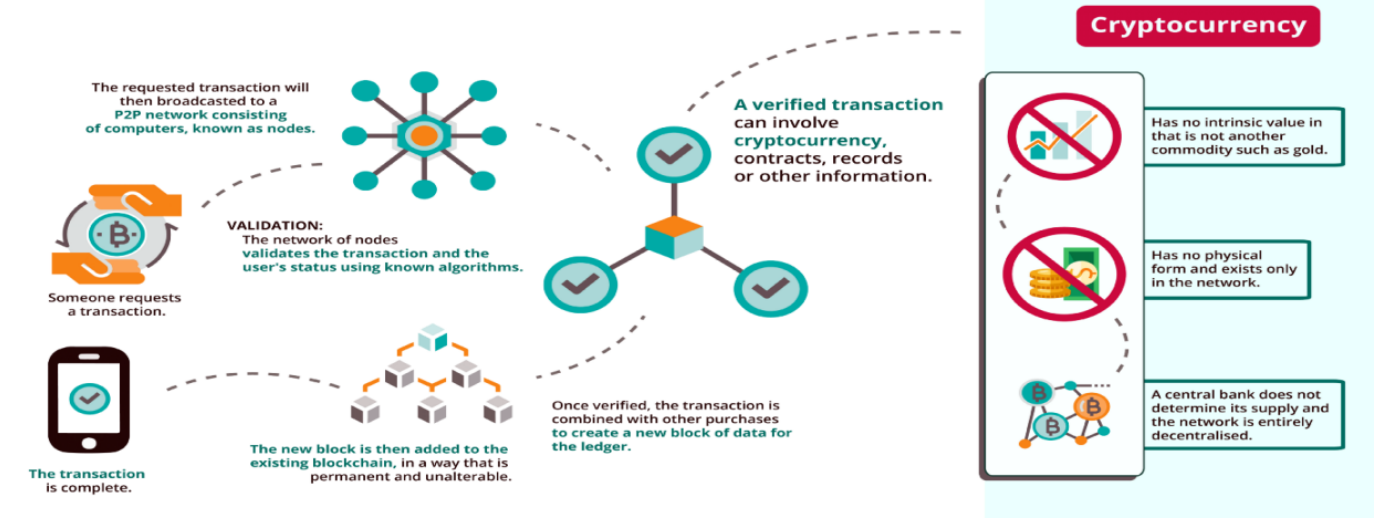

Fig 1: Blockchain mechanism[23].

\section{PROPOSED MOdeL}

In this section, a proposed commercial model is presented using blockchain. Figure 2 shows the proposed model, by which consumers are presumed to have a special record in a series, which contains a set of data as the personal information of the consumer, as well as a list of purposes for sale. Through which anyone reviews the sales list and his or her dates, added feedback option by consumers, and rating. 


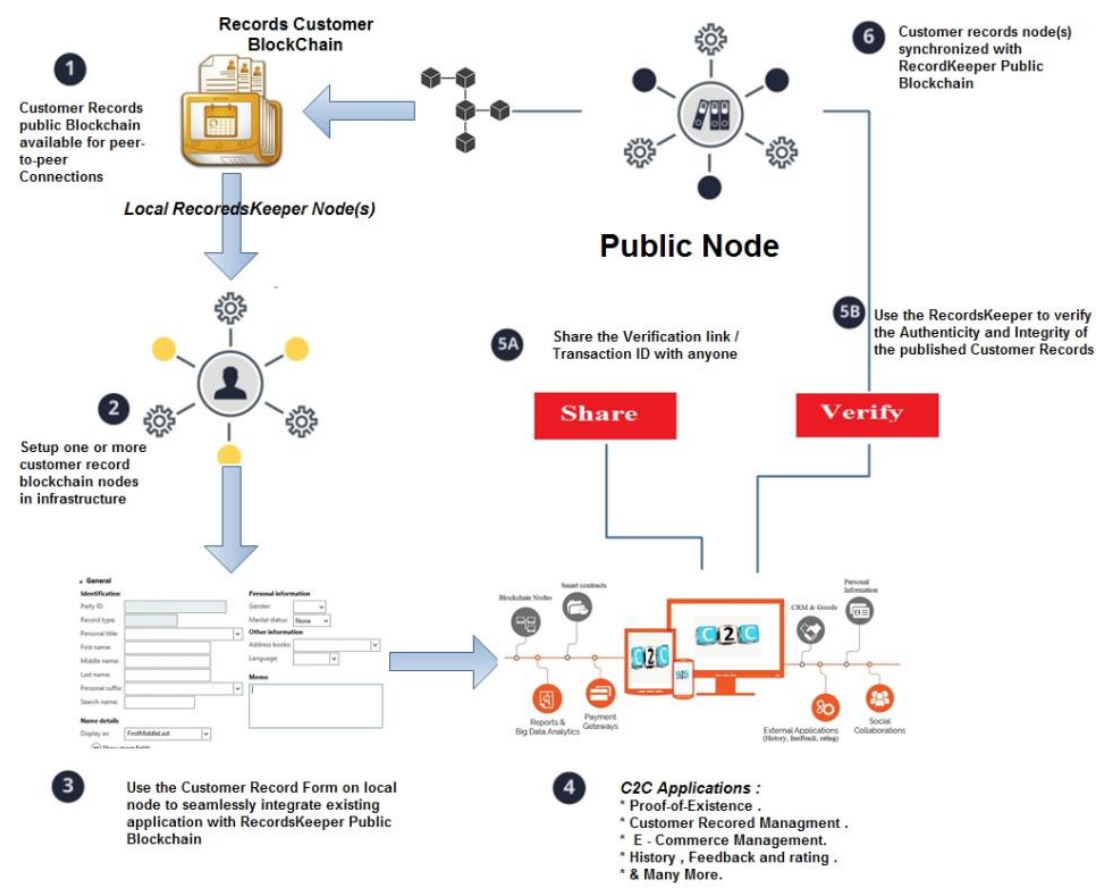

Fig 2:The Proposed Model

As shown in figure 3, applying the Blockchain Mechanism ine-commerceworks with the updating and distribution of data continuously to all users depending on the establishment of smart contracts to formalize business processes between consumers, in addition to the possibility of contracting in a way that can be achieved in an easy and effective manner.

We need three important factors to put blockchain in implementation, include Decentralization which means all the customers records should be distributed for all participates, you can make a transaction any time everywhere but under control. the second is transparency to make all the required data is visible, with no need the private data. The third is the immutability in the whole data that are entered into blockchain, the changes not allowed. To confirm those phases will use smart contracts are created for business transactions and are recorded in records, and encrypted and linked to the blockchain, and distributed to the rest of the users.

With feedback and evaluation, which is an important factor in this research, consumers can write their feedback after the completion of the sale and receipt of goods and then the appropriate assessment.Showing the details of the goods guarantees the credibility of the product. 


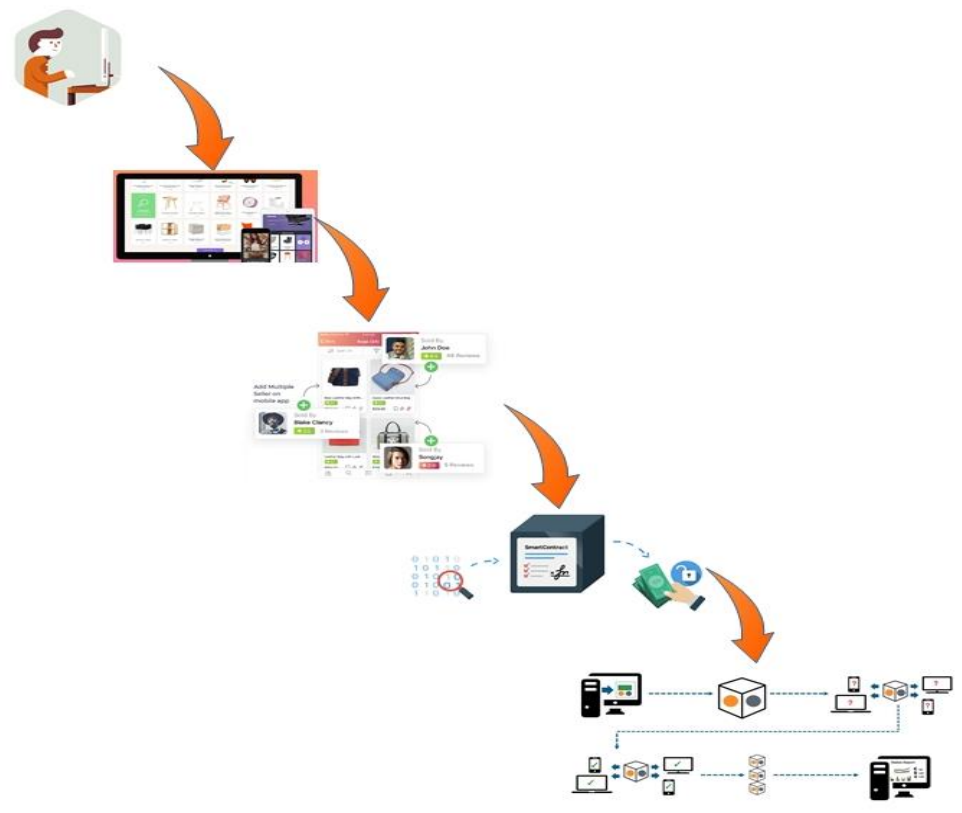

Fig 3 Ecommerce steps using the blockchain

Figure 4 below illustrates the sequence of a buying and selling process between consumers; as evident from the form, the process starts from displaying a consumer to his own product, which is owned by him with all the details related to the sale. Then any consumer who wishes to buy calls the seller's record to know the date and then proceeds to complete the sale and payment process, it is documented in the block and linked to the chain which considered a transfer of ownership. Then the transfer contract is distributed to all users; the step following the transfer process, feedback, and that through which the buyer writes feedback and uses smart contracts, and puts an evaluation of the product, which is distributed to all users.

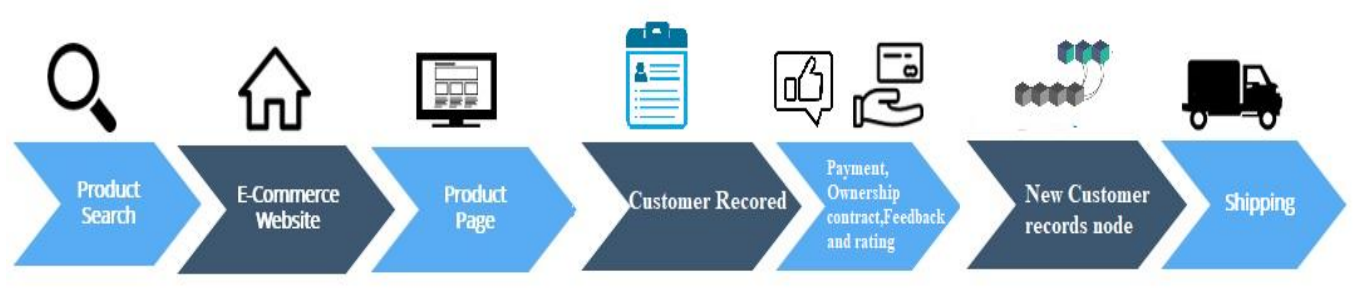

Fig 4: the sequence of a buying and selling process

\section{DisCUSSION}

This section will discuss the proposed model by tracking a hypothetical scenario asconsumer-toconsumer and focusing on replacing the traditional buying and selling process with the Blockchaintechnology.Consumerprofile, which contains information about the consumer, this profile,is distributed to all users of Blockchain. 
International Journal of Managing Information Technology (IJMIT) Vol.11, No.2, May 2019

As well as consumer feedback,Resulting in the interaction with the consumerfrom participants, the quality of the feedback is true, in addition to the data distributed to all users of Blockchain so that it is difficult to manipulate and create false information to the feedback. Then, dealing with Blockchain in the e-commerce process adds credibility to business processes to prevent fraud or tampering if the file is stored in the database, but the distribution gives some caution by displaying consumers with credibility without tampering it. Through the samescreen, anyone can decide according to the information dealer's feedbackto deal with the consumer or not.

The feedback mechanism for Blockchainconsumers, which only allows dealing with a unified feedback model, and through this option, feedback, cannot be accepted through a single screen to prevent manipulation or cheating feedback; and this screen appears in the completion of the process, sale and purchase, and shall be informed by two parties. Through the screen, you can also give a rating as is a custom in electronic commerce in the form of stars.

Blockchain and consumer-to-consumertrading increases the credibility of business processes by leveraging on the advantages of blockchain by distributing data to all subscribers and monitoring consumer behaviour. Blockchain, expected to become an effective alternative to the credibility and non-manipulation of transactions, becomes a successful competitor to the famous electronic business sites and becomes a strong competitor to entrepreneurship, which guarantees everyone to trade without bowing to the aspirations and monopoly of the third party. This forces small clients to deal through their gates, which takesa percentage of profits only to use their platforms [24].

C2C market is expected to grow on blockchain technology in the future because of its costeffectiveness. The cost of using blockchain in business transactions between consumers does not require additional fees by a third party to complete the transaction or to give confidence in transactions that is usually covered by large business companies [25].In addition to increasing business volume between consumers.

By using blockchain in a $\mathrm{C} 2 \mathrm{C}$ business, effective mechanisms have been created to achieve quality control and payment guarantees which are detailed and distributed to all participants, so that any user can follow the business transactions they would like to deal with, to create a genuine trust betweenconsumers through the feedback detection and evaluation, as well as the possibility of finding easy ways to make payments [26]. Blockchain trusting environment allows consumers to settle payments and is looking to increase business dealings and create jobs for anyone who wants to retail, without having to pay extra fees to brokerage firms used by consumers on their online platforms [27]. The possibility of confirming the quality and details of the goods offered for sale through the product record, which contains all the needed information, which is in the smart contract form, through the feedback of the goods can match details with the real goods sold and received; this imposes a new kind of valuation to prove the credibility of the consumer.

Transparency, trust,and ethics are considered as imperative elements by the consumer. In addition, many consumers make purchasing decisions based on the information stored in the blockchain. It allows producers to trackthe products from the moment of entraining the sales and purchase chain until the product is shipped to the consumer.

Blockchain is a true competitor to e-commerce companies, which will lose manyconsumers replaced by the blockchain as an effective and successful alternative to complete e-business operations. And it createsa trustful environment between consumers without the need for a third 
International Journal of Managing Information Technology (IJMIT) Vol.11, No.2, May 2019

party to establish the credibility and confidence of business transactions, as well as focus business processes on the sale and purchase without the need to pay percentages of profits to anyone.

\section{CONCluSion AND Future Work}

The technology of Blockchain and its application to the consumer-to-consumertrade is considered as a significant progress in e-commerce. It creates an environment of trust and credibility in trade transactions by tracking and distributing consumer records as well as, enabling everyone to see the progress of business transactions. As well as reading the feedback of consumers who wish to sell their needs. In this model, three smart contracts are connected to business processes between consumers (buy, sell, and transfer, details of goods, feedback,and evaluation); blockchain revolution is expected to be a successful or parallel alternative to the traditional process. It encourages small traders to carry out their operations without commercial platform interventions. The proposed model combines the idea of a $\mathrm{C} 2 \mathrm{C}$ and a blockchain technology in order to reach an effective trading environment for the success of this type of trade and to mitigate worriesbetween consumers. The model tried to create an environment of trust and credibility among consumers through the possibility of tracking their records, and giving the consumer the freedom to make decisions in dealing with the consumers of others by tracking their rates and feedback, depending on previous business transactions. It is expected to increase the commercial operations of the sale and purchase between consumers, and find alternative ways of payment through either installation or swap, and this is the result of confidence resulting from the model proposal.

This research can contribute significantly to the literature of advanced technology and ecommerce which needs applied research as a reference for $\mathrm{C} 2 \mathrm{C}$ to cover different areas of $\mathrm{E}$ commerce operations that support the conversion of traditional operations into digital operations. The benefits of blockchain technology and smart contracts are obtained as the expected future tools of e-commerce. Future work will include a detailed verification of technical aspects and remittances. In addition, a real scenario will be tested with the scrutiny of e-commerce experts.

\section{REFERENCES}

[1] Nanehkaran, Y.A (2013). An introduction to electronic commerce. International journal of scientific \& technology research, 2(4). Retrieved from http://www.ijstr.org/final-print/apr2013/AnIntroduction- To-Electronic-Commerce.pdf.

[2] Ohidujjaman, Hasan, M. \& Huda, M.N. (2013). Ecommerce Challenges, Solutions and Effectiveness Perspective Bangladesh. International Journal of Computer Applications, 70(9).9-17. Retrieved from http://icdst.org/pdfs/files/5f540f8a517ec822aaba2ad7869dcdec.pdf.

[3] Gunasekaran, A., et al. "E-commerce and its impact on operations management."International journal of production economics 75.1 (2002): 185-197.

[4] Lifang Peng, Zhong Chen, Qi Li, "Model and Method for Evaluating Creditability of C2C Electronic Trade", ICEC'06, August 2006.

[5] Kim, T. Y., Dekker, R., \& Heij, C. (2017). Cross-border electronic commerce: Distance effects and express delivery in European Union markets. International Journal of Electronic Commerce, 21(2), $184-218$. 
International Journal of Managing Information Technology (IJMIT) Vol.11, No.2, May 2019

[6] Egger, F. and B. de Groot. (2000). "Developing aModel of Trust for Electronic Commerce: An Application to a Permissive Marketing Web Site." In Proceedings of the 9th International WorldWide Web Conference Foretec Seminars.

[7] Hall, L. L., Paso, E., López, F. J., \& College, M. S. (2008). Measuring E-Commerce Technology Enabled Business Value: An Exploratory Research. International Journal of E-Business Research, $4(2), 48-68$.

[8] Huang, Z., \& Benyoucef, M. (2012). From E-commerce to Social Commerce: A Close Look at Design Features. Electronic Commerce Research and Applications.

[9] Dylan Yaga,Peter Mell,Nik Roby,Karen Scarfone,(2018).Blockchain Technology Overview.NISTIR 8202.https://doi.org/10.6028/NIST.IR.8202.

[10] K. Christidis and M. Devetsikiotis, "Blockchains and smart contracts for the internet of things," IEEE Access, vol. 4, pp. 2292-2303, 2016.

[11] C. Natoli and V. Gramoli, "The blockchain anomaly," in 15th International Symposium on Network Computing and Applications (NCA), 310-317, IEEE, 2016.

[12] Clemons, E.K. An empirical investigation of third-party seller rating systems in e-commerce: The case of buySAFE. Journal of Management Information Systems, 24, 2 (2007), 43-71.

[13] Pradnya B. Rane, Dr. B.B.Meshram. "Transaction Security for Ecommerce Application" IJECSE ISSN2277-1956. 2012.

[14] Hall, L. L., Paso, E., López, F. J., \& College, M. S. (2008). Measuring E-Commerce Technology Enabled Business Value: An Exploratory Research. International Journal of E-Business Research, $4(2), 48-68$.

[15] Peck, Morgen E. "Do You Need a Blockchain?" IEEE Spectrum: Technology, Engineering, and $\begin{array}{lllll}\text { Science } & \text { News, } & \text { IEEE } & \text { Spectrum, }\end{array}$ https://spectrum.ieee.org/computing/networks/do-you-need-a-blockchain.

[16] Q. K. Nguyen, "Blockchain-A Financial Technology for Future Sustainable Development," in Green Technology and Sustainable Development (GTSD), 2016, pp. 51-54.

[17] Y. Yuan and F. Y. Wang, "Towards blockchain-based intelligent transportation systems," in 2016 IEEE 19th International Conference on Intelligent Transportation Systems (ITSC), 2016, pp. $2663-$ 2668.

[18] S. Nakamoto, "Bitcoin: A peer-to-peer electronic cash system," 2008.

[19] M. Mettler, "Blockchain technology in healthcare: The revolution starts here," in e-Health Networking, Applications and Services (Healthcom), 2016 IEEE 18th International Conference on, 2016, pp. 1-3.

[20] Reid, F. and Harrigan, M. An analysis of anonymity in the bitcoin system. In Proceedings of the Third IEEE International Conference on Social Computing (Boston, MA, Oct. 9-11). IEEE Press, 2011, $1318-1326$.

[21] X. Xu, C. Pautasso, L. Zhu, V. Gramoli, A. Ponomarev, A. B. Tran, and S. Chen, "The blockchain as a software connector," in 2016 13th Working IEEE/IFIP Conference on Software Architecture (WICSA), pp. 182-191, IEEE, 2016. 
International Journal of Managing Information Technology (IJMIT) Vol.11, No.2, May 2019

[22] G. Karame, “On the security and scalability of bitcoin's blockchain,” in Proceedings of the 2016 ACM SIGSAC Conference on Computer and Communications Security. ACM, 2016, pp. 1861-1862.

[23] Medium, https://medium.com/@imarticus/infographics-what-is-blockchain-6a1f93e64e79

[24] Mahdi H. Miraz, Maaruf Ali ,(2018).Applications of Blockchain Technology beyond Cryptocurrency,Annals of Emerging Technologies in Computing (AETiC), Print ISSN: 2516-0281, Online ISSN: 2516-029X, pp. 1-6, Vol. 2, No. 1, 1st January 2018, Available: http://aetic.theiaer.org/archive/v2n1/p1.pdf

[25] Yu Zhang and Jiangtao Wen,(2017).The IoT electric business model: Using blockchain technology for the internet of things,Peer-to-Peer Networking and Applications, July 2017, Volume 10, Issue 4, pp 983-994

[26] Zott C, Amit R (2008) The fit between product market strategy and business model: implications for firm performance[J]. Strateg Manag J 29(1):1-26

[27] Hemang Subramanian ,(2018).Decentralized Blockchain-Based Electronic Marketplaces,Communications of the ACM, Vol. 61 No. 1, Pages 78-84,10.1145/315833. 